\title{
Network-Adaptive QoS Routing using local information
}

\author{
Jeongsoo Han \\ Department of Internet Information technique, \\ Shingu College, Korea \\ jshan@shingu.ac.kr
}

\begin{abstract}
In this paper, we propose the localized adaptive QoS routing scheme using POMDP(partially observable Markov Decision Processes) and Exploration Bonus. In order to deal with POMDP problem, we use CEA(Certainty Equivalency Approximation) technique that involves using the mean value of random variables, since it is intractable to determine the optimal solution. And we present a new path selection using the Exploration Bonus method in order to find detour path, which is better than current path and more adaptive to network environment. Also we evaluate performances of service success rate and average hop count which varies with $\phi$ and $\kappa$ performance parameters, which are defined as exploration count and intervals.
\end{abstract}

Keywords: Localized Adaptive QoS Routing, Reinforcement Learning, POMDP, Exploration Bonus, Certainty Equivalency Approximation, Edge-disjoint multi-path

\section{Introduction}

In localized routing technique, each node in the network makes local routing decisions based just on local information, i.e., information on the status of the network that has been obtained from the neighbor nodes. Also, routing policies aim to minimize the average overall blocking probability based on local information. If local information is used, it is difficult to determine how a routing decision made on a single node may influence the network's overall performance. It has been demonstrated that localized QoS routing is simple, stable, adaptive and effective in comparison to global QoS routing schemes[1]. [2,3] have proposed a localized QoS routing scheme, proportional sticky routing (psr). The psr scheme maintains self-adaptivity by using the maximum permissible flow blocking parameter and controlling the number of flows routed along a path in each cycle and by re-adjusting flow proportions after every observation period. But, this approach has three main drawbacks. Firstly, the traffic pattern is always unknown in practical networks. Secondly, we need to know the precise information along the path to calculate the blocking probability. Thirdly, even though we are able to identify the traffic pattern and calculate the blocking probability, the iteration time needed to solve the global optimization problem can 
be significant[4]. To overcome the above problems, [4] proposes a reinforcement learning(RL) based scheme(Q-Learning) to make path selection in the absence of network traffic pattern and global information in the core networks.

In general, an environment of RL is formalized as MDP(finite-state Markov Decision Process), which calculates the long term values of state action pairs using a temporal difference method for approximating solutions to dynamic programming. But a current optimal action is not guaranteed at future because environments connected with agent can change stochastically over time. In realworld environments, it will not be possible for the agent to have perfect and complete perception of the state of the environment. Unfortunately, complete observability is necessary for learning methods based on MDPs. In this paper, we consider the case in which the agent makes observations of the state of the environments, but these observations may be noisy and provide incomplete information. We will consider extensions to the basic MDP framework for solving partially observable problems. The resulting formal model is called a partially observable Markov Decision Process or POMDP, which starts from a probabilistic characterization of the uncertainty the agent has about the world. Performing dynamic programming to solve a POMDP is highly computationally expensive, and is intractable to determine the optimal solution. Therefor,e we make a form of CEA(Certainty Equivalence Approximation), in which the stochasticity in the world is simplified through the use of expectations. In this paper, we adapt these techniques to routing problems because network environments can change stochastically over time and in absence of global information on each node. We provide a localized routing technique as using POMDP with CEA and a new way of path selection using the exploration bonus method which is more adaptive to network environments. For this, this paper presents the multi-path searching algorithm for the shortest pairs of edge-disjoint paths that revised [10]. Also we evaluate performances of service success rate and average hop count relevant with and performance parameters, which are defined as exploration count and intervals. The organization of this paper is as follows. In chapter 2, we show the proposed routing techniques, updating routing information rules using POMDP with CEA and decision making process with exploration bonus and the multipath searching algorithm for the shortest pairs of edge-disjoint paths. This is followed by the presentation of performance evaluation in chapter 3 . We conclude the paper in chapter 4 .

\section{Proposed Algorithm}

In localized routing technique, each node in the network makes local routing decisions based just on local information. From this viewpoint, it can be connected with POMDP problem which its agent ignores about its environments. That is, that the agent knows a) its own state $x^{t}(n) \in X$ at all times $n$ within trial $\left.t, \mathrm{~b}\right)$ the costs $C_{x}(a)$ of taking the actions, c) the states that are absorbing, and d) the distribution governing the way the transition probabilities change. But, the 
agent does not know transition probabilities and its knowledge as to how they change. This ignorance turns a Markov decision problem into a POMDP.

\subsection{Routing Model based on POMDP}

In order to obtain a proposed network routing model usefully, it is possible to make that the following network's elements correspond to the basic elements of a POMDP : an agent(node which receives a request), the set of actions available to the agent $(A$, a set of predefined paths to a destination), the state set $(X$, each network node), a value iteration $\left(V_{a}^{t, n}(x, y)\right.$, at time $n$ during trial $t$, the local routing policy which state $x$ for destination node $y$ should be choose). Also, because a source node is ignorant of information about network environments, let $p_{x y}^{t}(a)$ be a routing success probability(ie, transition probability) when a source node(state $x \in X$ ) takes a predefined path(action $a \in A$ ) to destination node(state $y \in X$ ), where $t$ is the trial number. The network environments are non-stationary in the sense that between routing trials, the transition probabilities can change in a Markovian manner, according to a probability distribution $U\left[P_{x y}^{t+1}(a) \mid P_{x y}^{t}(a)\right]$ (belief state). The agent maintains a distribution over them which it updates based on Bayes's rule in the light of information collected from the environment, uses it for routing decision on state. Therefore, the agent can infer the unknown transition probabilities $p_{x y}^{t}(a)$ through the distribution over the transition probabilities. And $C_{x y}(a)$ is hop count from state $x$ to destination $y$ when taking action $a$. Performing dynamic programming to solve a POMDP is highly computationally expensive, and therefore we make a form of CEA, in which the stochasticity in the world is simplified through the use of expectations $\left(q_{x y}^{t, n}(a)=E_{\gamma^{t, n}}\left[p_{x y}(a)\right]\right)$ instead of all the $p_{x y}^{t}(a)$, where $\gamma^{t, n}\left[p_{x y}(a)\right]$ is probability distribution at time $n$ during trial $t$. Fig. 1 shows the network environments using POMDP and CEA techniques in this paper.

Under the approximation, the agent performs value iteration in the assumed mean process as follows :

$$
V_{\alpha+1}^{t, n}(x, y)=\min _{a \in A}\left\{C_{x y}(a)+\gamma \Sigma_{y \in X} q_{x y}^{t, n}(a) V_{\alpha}^{t, n}(x, y)\right\}
$$

where $0 \leq \gamma \leq 1$ is discount factor and $\alpha$ is the number of the dynamic prograaming iteration.

\section{$2.2 \quad$ Updating Routing Information Rules}

The course of updating routing information is as follows : Once the agent picks one of the possible actions that minimizes the right-hand side of equation (1), performs it, uses Bayes' rule to compute the posterior distribution over the transition probabilities based on the transition actually observed, and calculates $q_{x y}^{t, n+1}(a)$. The agent attempts to take such an action for admitting a request with certain bandwidth requirement if there is enough bandwidth in the chosen action, so it is possible to route a request on that action. In this paper, we define that such an action is termed as effective action and is defined as $e_{x y}^{t, n}(a)=1$. 


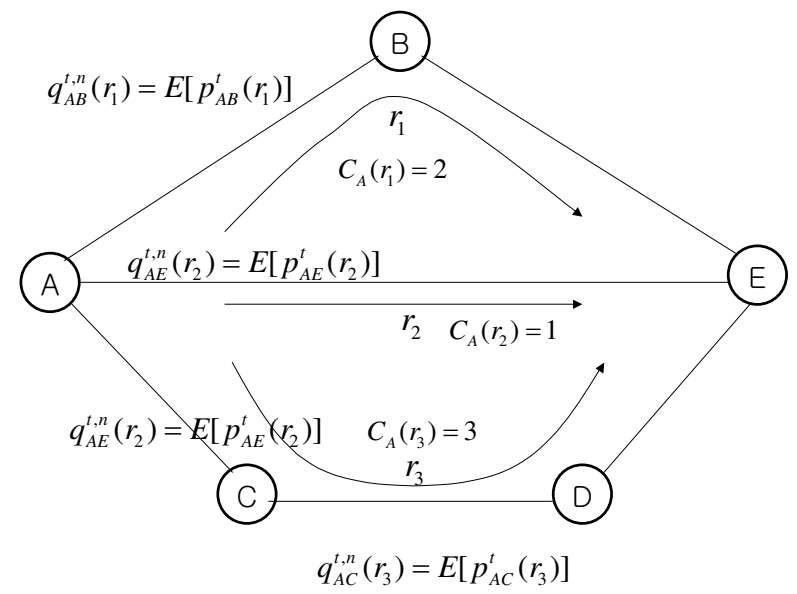

Fig. 1. The network environments using POMDP and CEA techniques

And if otherwise, ineffective action and $e_{x y}^{t, n}(a)=0$. In this paper, the agent does not know $e_{x y}^{t, n}(a)$, ie it does not know whether it is possible to route on such an action because it does not absent of global information. Therefore, we define a probabilistic model $(\phi)$ of the efficacy of the transitions ie, the probabilities of routing success on each path. Specifically, let $q_{x y}^{t, n}(a)$ be the agent's estimate of the efficacy of action $a$ at $x$ for $y$ at time $n$ during trial $t$. And the agent assumes that between each trial with some small arrival rate $(\kappa)$, each $e_{x y}^{t, n}(a)$ gets set to a new probability value, independent of its previous value. After trial $t-1$, the updating routing information at $x$ occurs as follows:

$$
q_{x}^{t, 0}(a)= \begin{cases}\kappa \phi+(1-\kappa) q_{x}^{t-1,0}(a) & (2-1) \\ 1-\kappa(1-\phi) & (2-2), \\ \kappa \phi & (2-3)\end{cases}
$$

When $a$ was not tried at $x$ during the trial, we can define as (2-1). When $a$ was tried at $x$ and was successful, we also define as (2-2). And (2-3) means when $a$ was tried at $x$ and was unsuccessful. $q_{x y}^{t, n}(a)$ is reset when the agent tries $a$ at $x$ to whatever actually happened. For actions that were not attempted, $q_{x y}^{t, n}(a)$ relaxes towards $\phi$ at a rate governed by the arrival rate $\kappa$. And others cases, $q_{x y}^{t, n}(a)$ is reset as each values.

\subsection{Decision Making Process(Exploration Bonus)}

The decision-making process consists of selecting the path through which the request will be connected. The agent's initial uncertainty about the transition or reward structure of the world should drive the initial experimentation, and, if the world can change stochastically over time, then this further source of uncertainty should drive continuing exploration. Unfortunately, it is computationally 
intractable for the agent to determine the correct experiments even if it knows what it does not know. And in using this mean process, the approximation fails to directly account for the fact that if a transition on the way to the destination is blocked because of QoS problem, then the agent will have to choose some other path to that destination. To solve this problem in this paper, we shows the heuristic algorithm, exploration bonus method which is added to the immediate value of a number that is a function of this how long it has been since the agent has tried that action in that state. Therefore, rather than have the agent model the efficacy of transitions, we had it directly add to the immediate cost for a move an exploration bonus of $\alpha \sqrt{n_{x}(a)}$ for trying action $a$ at state $x$, if that action had not been tried for $n_{x}(a)$ trials.

Table 1. Shortest Edge-disjoint Multi-path Searching Algorithm

\begin{tabular}{|ll|}
\hline Initialization] & Let $\delta=\phi$ be the desired path set. \\
Step 1 & [Computation of shortest path] \\
& We obtain the shortest path tree $T$ rooted at source $s$ using Dijkstra's \\
& algorithm. \\
& Let the shortest path from $s$ to destination $v$ be denoted as $P_{1}$ and the \\
& cost of the shortest path from $s$ to each vertex $x$ be denoted by $C(s, x)$. \\
& {$[$ Cost transformation and Modification of graph] } \\
Step 2 & The cost of every edge $(a, b)$ in $G$ is transformed to $c^{\prime}(a, b)=c(a, b)+$ \\
& $C(s, a)-C(s, b)$. \\
& All the edges belonging to $T$ will have a reduced cost equal to 0 due to \\
& this transformation. \\
& And reverse the orientation of all edges of $G$ which lie in $P_{1}$ to form \\
& a new graph $G_{v}$. \\
& {$[$ Computation of shortest path] } \\
& Compute the shortest path from $s$ to $v$ in $G_{v}$. Let it be denoted as $P_{2}$. \\
Step 3 & {$[$ Shortest path pair Generation] } \\
Step 4 & The desired path pair, say $P_{1}^{\prime}$ and $P_{2}^{\prime}$ is obtained by $\left\{P_{1} \cup P_{2}\right\}-$ \\
& $\left\{P_{1} \cap P_{2}\right\}$. \\
& And compute $\delta=\left\{P_{1}^{\prime}, P_{2}^{\prime}\right\}+\delta$. \\
& {$[$ Reduction of graph] } \\
& We generate new graph $G^{\prime}$ by deleting all edges belonging to $\delta$ from $G$. \\
Step 5 & And let $G=G^{\prime}$. \\
& {$[$ Repeat] } \\
& We repeat the previous Steps from 1 to 5 until there are no edges \\
& connected with $s$.
\end{tabular}




\subsection{Shortest Edge-disjoint Multi-path searching algorithm}

In this paper, we propose a SEMA(Shortest Edge-disjoint Multi-path searching Algorithm) which revises a SSP(Single-Sink Problem) algorithm that S.Banerjee proposed [10] and is used for routing on POMDP routing model This algorithm of finding multiple disjoint path from source to a destination can be formulated as a the minimum-cost flow problem and solved by successive iterations of Dijkstra's shortest path algorithm. Let $G=(V, E)$ be a directed graph having a nonnegative cost $c(u, v)$ as associated with each edge $(u, v) \in E$ and let $|V|=n$ and $|E|=m$. The SEMA is as follows Table 1.

If the upper bound of an efficient implementation of Dijkstra's algorithm is denote by $O(n, m)$, SEMA can also be solved in $O(n, m)$ time sequentially.

\section{$3 \quad$ Simulation and Results}

This section illustrates how the amount of exploration depends on the performance parameters $\phi$ and $\kappa$ using POMDP, CEA algorithms that adapted Exploration Bonus. For this, we will provide the test environments as follows.

\subsection{Simulation Environments}

As seen in Fig. 2, we use two test network for simulation, and conduct out experiment under the network environment of [1] and the request requirement of [2]. All the links are assumed to be bi-directional and of the same capacity, with $C$ units of bandwidth in each direction. Flows arriving into the network are assumed to require one unit of bandwidth. Hence each link can accommodate at most $C$ flows simultaneously. The flow dynamics of the network are modeled as follows. Flows arrive at a source node according to a Poisson process with rate $\kappa$. The destination node of a flow is chosen randomly from the set of all nodes except the source node. The holding time of a flow is exponentially distributed with mean $\frac{1}{\mu}$. The offered network load is given by $\rho=\frac{k N h}{\mu L C}$ where $N$ is the number of source nodes, $L$ is the number of links and $h$ is the mean number of hops per flow, averaged across all source-destination pairs. The parameters used in this simulation are $C=20, h=2.36$ and $\frac{1}{\mu}=60$ seconds. We also use multi-paths that generated by SEMA as feasible paths for requests. When we evaluate the Exploration Bonus method, we use the greedy policy until blocking happens and then apply the Exploration Bonus method.

\subsection{Results Analysis}

The agent's experience was divided into a number of routing trials on feasible path. In each trial, the agent starts at the source node and takes actions until it reaches the destination node. In all cases, complete synchronous value iteration was performed before each step in each trial using equation (1) to convert the current model of the world into a suggested action. The value function produced 


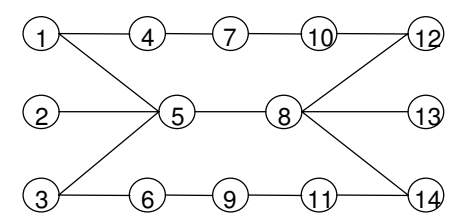

(a) Simple Test Network

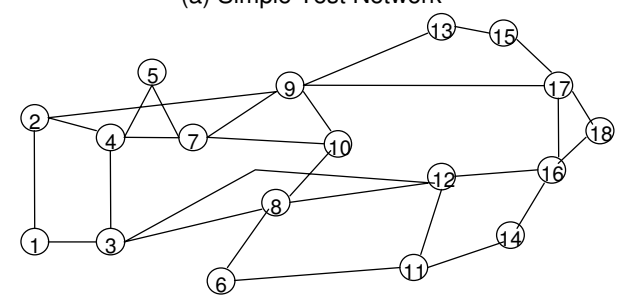

(b) The ISP Network

Fig. 2. The Test Networks

at the previous step was used to initialize value iteration, so convergence took only a few iterations unless the agent found out in the previous step that the world was substantially different from what it expected. The model was updated in the course of a trial as the agent discovered that transitions were effective or ineffective. At the end of a trial, all the expected efficacies $q_{x}^{t, n}(a)$ were updated to take account of the chance that each transition might have been changed.

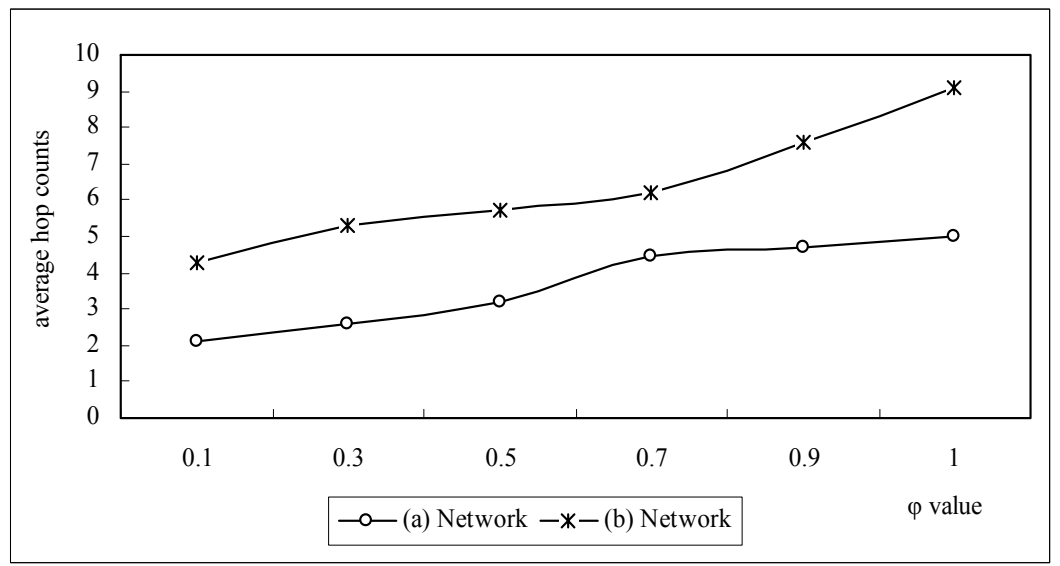

Fig. 3. The performance of routing average hop count on test network which varies with $\phi$ value

Fig. 3 shows the performance of routing average hop count on each test network which varies with a $\phi$ value. As $\phi$ gets bigger, the system will always 


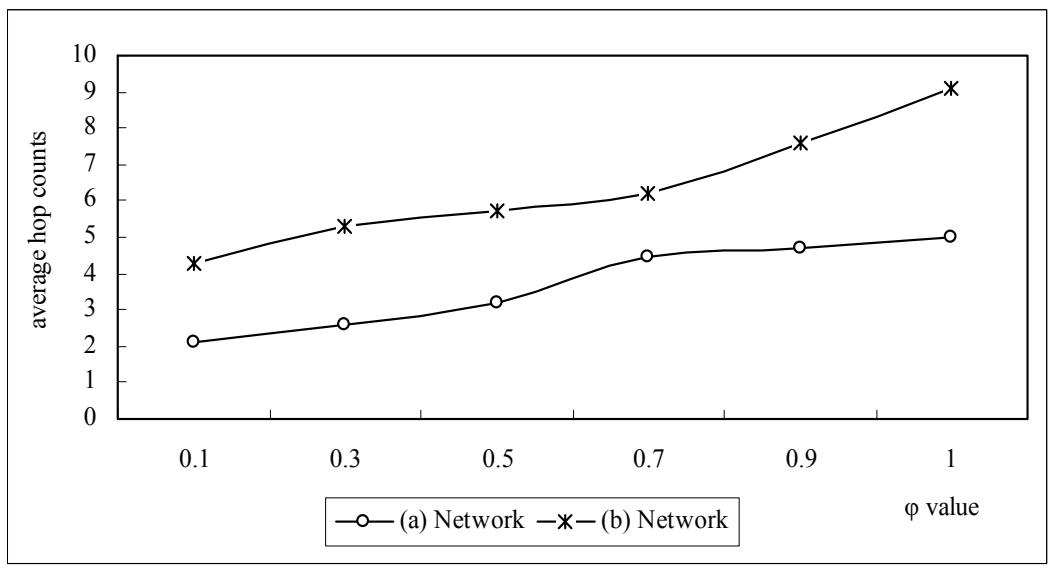

Fig. 4. The performance of routing success rate on test network which varies with $\phi$ value

eventually explore to find paths that might be longer than its current one - ie it will find the detours when the current path will be blocked because of various QoS parameters. But as $\phi$ gets smaller, the system becomes more pessimistic. It will not bother to find large detours to try possibly better paths. Therefore, the lower $\phi$, the more restricted the excursions. As seen in Fig. 3, the actual average length of the shortest paths also increased(from 3.5 hop counts at the real value of $\phi=0.2$ to 5 hop counts at the real value of $\phi=0.9$ in the Fig. 2 (a) test network). It is attributed to the fact that it will find detours other than the current path as $\phi$ increases ( $\kappa$ was fixed at 0.03 and was known to the agent). Also the Fig. 2 (b) test network that has relatively many links between the nodes has more hop counts than the Fig. 2 (a) test network.

Fig. 4 shows the performance of routing success rate on each test network which varies with a $\phi$ value. As seen in the result of Fig. 3, the more $\phi$ increases, the more detours other than the current path the agent explore. Therefore, the performance of routing success rate will be improved. Also $\kappa$ was fixed at 0.03 and was known to the agent.

Fig. 5 shows the performance of routing success rate on each test network which varies with a $\kappa$ value. As indicated above, the other parameter $\kappa$ also has an effect on the relative amounts of exploration and exploitation, but in a more indirect way. Whereas $\phi$ determines the ultimate amount of exploration about the current best-known path, $\kappa$ controls how soon that exploration happens. Therefore, the more frequent explorations, the higher service success rate because it will be clear that increasing $\kappa$ increased the amount of exploration. $\phi$ was fixed at 0.5. As seen in Fig. 5, the actual average success rate also increased (from $38 \%$ at the real value of $\kappa=0.03$ to about $50 \%$ at the real value of $\kappa=0.05$ in the Fig. 2 (b) test network). It is attributed to the fact that the amount of exploration will increase. 


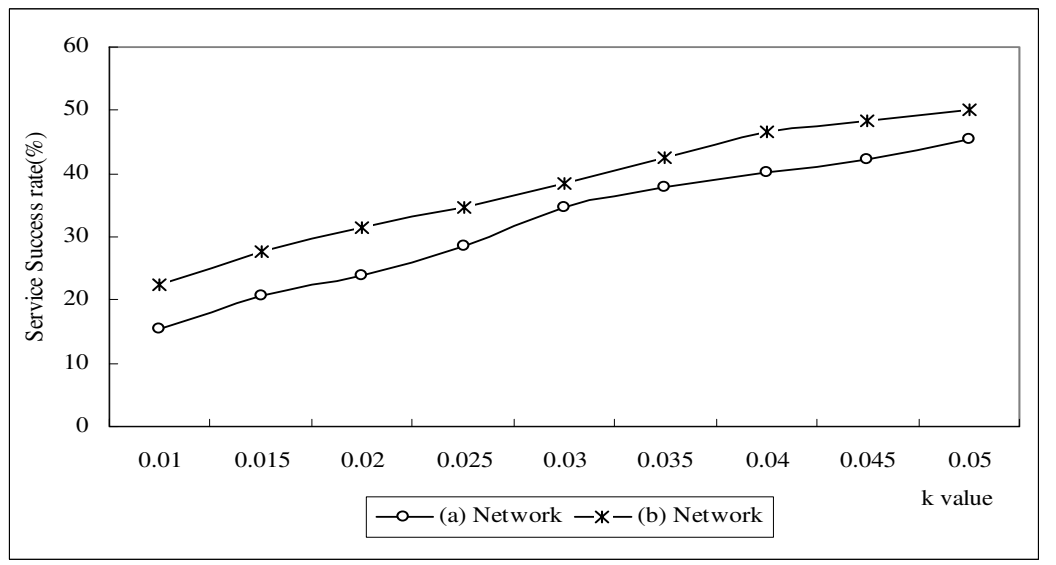

Fig. 5. The performance of routing success rate on test network which varies with $\kappa$ value

\section{Conclusion}

In this paper, we present the new Localized Adaptive QoS routing technology without the knowledge of network global information. For this, we proposed the POMDP model and CEA technology that provide a better QoS routing method using only local information on source nodes and provide a much more adaptive way of path selection depending on a network environment. We also proposed an edge-disjoint multi-path searching algorithm, SEMA. As with POMDPs, we start from a probabilistic characterization of the uncertainty the agent has about the network environment, apply this model to the Localized QoS routing method. And we make a use of CEA, which use the mean values of random variables, because performing dynamic programming to solve a POMDP is highly computationally expensive and is intractable to determine the optimal solution. For better path selection, we propose Exploration Bonus because network environment connected with agent can change over time, so a current optimal action is not guaranteed at future. Also we evaluate performances of service success rate and average hop count which varies with $\phi$ and $\kappa$ performance parameters, which are defined as exploration count and intervals. As a result, whereas the parameter $\phi$ determines the amounts of exploration to find a better path than the current one, $\kappa$ controls how soon that exploration happens. As $\phi$ gets bigger, the system will always find the detours when the current path will be blocked because of various QoS parameters. But as $\phi$ gets smaller, the system becomes more pessimistic. It will not bother to find large detours to try possibly better paths. Therefore, if $\phi$ gets closer to 1 , the success rate and the average hop counts will get higher. Also, the higher $\kappa$, the higher service success rate because the amounts of exploration will increase. 


\section{References}

1. X.Yuan and A.Saifee, "Path Selection Methods for Localized Quality of Service Routing", Technical Report, TR-010801, Dept of Computer Science, Florida State University, July, 2001

2. Srihari Nelakuditi, Zhi-Li Zhang and Rose P.Tsang, "Adaptive Proportional Routing: A Localized QoS Routing Approach", In IEEE Infocom, April 2000.

3. Srihari Nelakuditi, Zhi-Li Zhang, "A Localized Adaptive Proportioning Approach to QoS Routing", IEEE Communications Magazine, June 2002

4. Y.Liu, C.K. Tham and TCK. Hui, "MAPS: A Localized and Distributed Adaptive Path Selection in MPLS Networks" in Proceedings of 2003 IEEE Workshop on High Performance Switching and Routing, Torino, Italy, June 2003, pp.24-28

5. Yvn Tpac Valdivia, Marley M.Vellasco, Marco A. Pacheco "An Adaptive Network Routing Strategy with Temporal Differences", Inteligencia Artificial, Revista Lberoamericana de Inteligencia Aritificial, No 12(2001), pp. 85-91

6. Leslie Pack Kaelbling, Michael L. Littman, Andrew W.Moore, "Reinforcement Learning:A Survey", Journal of Artificial Intelligence Research 4, 1996, pp 237-285

7. P.Marbach, O.Mihatsch, and J.N.Tsitsiklis, "Call Admission Control and Routing in Integrated Service Networks Using Neuro-Dynamic Programming", IEEE Journal on Selected Areas in Communications, Vol. 18, No.2, Feb 2000, pp.197-208

8. Jeong-Soo Han, "A Localized Adaptive QoS Routing using $\operatorname{TD}(\lambda)$ method", Journal of Korean Institute of Communication and Sciences, Vol.30, No.5B, pp. 304-309, 2005

9. Gregory Z. Grudic, Vijay Kumar, "Using Policy Gradient Reinforcement Learning on Automous Robot Controllers", IROS03, Las Vagas, US, October, 2003 [11] Richard S. Sutton etc, "Policy Gradient Methods for Reinforcement Learning with Function Approximation", Advances in Neural Information Processing System, pp. 1057 1063, MIT Press 2000

10. S.Banerjee, R.K. Ghosh and A.P.K Reddy, "Parallel algorithm for shortest pairs of edge-disjoint paths", J.Parallel Distrib. Comput. 33(2):165-171 (1996) 